\title{
Removal of Some Pesticides from Contaminated Water Using Low Cost Materials
}

\section{${ }^{1}$ Mokhtar S. Beheary, ${ }^{2}$ Fatma A. El-Matary, ${ }^{3}$ Hazem T. Abd El- Hamid, ${ }^{3}$ Abdel Rahman Al-Tawaha}

${ }^{1}$ Environmental Science Department, Faculty of Sciences, Port Said University, Egypt.

${ }^{2}$ Marine Pollution Department, National Institute of Oceanography and Fisheries, Egypt.

${ }^{3}$ Department of Biological sciences, Al-Hussein bin Talal University, Maan, Jordan

Correspondence Author: Mokhtar S. Beheary, Environmental Science Department, Faculty of Sciences, Port Said University, Egypt..

Received date: 19 April 2018, Accepted date: 15 July 2018, Online, Online date: 31 July 2018

Copyright: (C) 2018 Mokhtar S. Beheary, et al. This is an open-access article distributed under the terms of the Creative Commons Attribution License, which permits unrestricted use, distribution, and reproduction in any medium, provided the original author and source are cre dited.

\begin{abstract}
The presence of pesticides in the environment is of high concern due to their persistent nature and chronic adverse effect on human health and the aquatic environment. In this study, efforts are made to develop low cost adsorbents to remove pesticides from aqueous solution. These materials have numerous advantages such as high sorption capacities, good modifiability and recoverability, insensitivity to toxic substances, simple operation in the treatment processes. Three types of adsorbents were used for removal of five kinds of pesticides from aqueous solution. The percent removal by using banana crust meal, biochare and spent tea leaves were: Atrazine (99.6\%, 92.4\% and 93.7\%), Diuron (96.7\%, 80.14\% and 82.3\%), Chloropyrifos $(61.8 \%, 72.9 \%$ and $73.4 \%)$, Dimethoate $(64.4 \%, 42.11 \%$ and $54.18 \%)$, and Imidacloprid $(77.9 \%, 93.09 \%$ and $96.9 \%)$, respectively. Banana meal has the ability to remove large amount of atrazine, diuron and dimethoate. Spent tea has the ability to remove chloropyrifos and Imidacloprid more than banana crust leaves and biochare.. Banana rust meal, biochare and spent tea leaves were found to be effective and low-cost alternative adsorbents for the removal of pesticides from aqueous solutions. The preparation method tolerates the usage of this material by local societies for active treatment of contamination.
\end{abstract}

Key words: pesticides, treatment, Low-cost biosorbents, water.

\section{INTRODUCTION}

The use of agrochemicals is essential in today's agricultural practice both in the developed and in the still developing countries. Using pesticides in agriculture to control some pests, as insects, weeds, and plant diseases, have been subject to considerable legislative, regulatory, and consumer scrutiny over the past few years [1]. The pesticides are being disseminated throughout the environment. The pesticides have many hazards such as; direct impact on humans, impact through food commodities, impact on environment, effect on beneficial soil microorganisms and soil fertility, contamination of air, soil, surface, groundwater and non-target vegetation [2]. Obviously, exposure to pesticides poses a continuous health hazard, especially in the agricultural working environment. By their very nature most pesticides show a high degree of toxicity because they are designed to kill certain organisms and thus create some risk of harm.

Within this context, pesticide use has raised serious concerns not only of potential effects on human health, but also about impacts on wildlife and sensitive ecosystems [3]. Their use must be controlled in order to avoid that unacceptable levels can be reached in different environmental compartments. In-field and laboratory experimental studies and monitoring surveys provide information on the leaching properties and on concentrations of many pesticides in groundwater [4]. Occurrence of pesticides in groundwater resources has grown in the past few years and has become an intensive and burning issue of discussion. Sorption is a major process controlling the fate of organic pollutants in all compartments of the environment. Pesticides sorption affects other processes like transport, leaching, degradation, volatilization, bioaccumulation, bioavailability which influence the final fate of these compounds in the soil environment [5].

Sorption effects pesticides leaching in the subsurface and has to be account when predicting pesticides transport [6]. The leaching of pesticides in soil as well as reaching groundwater is a considerable problem. The grade of reversibility in adsorption-desorption reactions is of importance for the evaluation of the mobility of pesticides in the aquatic environment [7]. Groundwater pollution affects the health of humans not only by used for drinking purpose, but also can act as a source of contamination for food chain, when used for irrigation [8]. Pesticides are a main type of nonpoint-source contaminants to groundwater, and their discharge to the nation's surface water may be a contributing factor toward the decline of the living resources and the deterioration of the ecosystems. A survey by the USEPA found pesticide detection in 16,606 wells in 45 states, with concentrations in 10,000 of these exceeding health advisory limits. Cost-effective assessment tools are needed to regulate the use of agricultural chemicals, identify areas which are potentially susceptible to nonpoint-source contamination, and support ecosystem restoration goals by improving the nation's water quality [9]. The concern of regulation authorities in the possible pollution of groundwater by pesticides begins from 1970s [10]. The prevention of groundwater contamination is cost effective than restoring polluted aquifers. For that very reason it is of maximum interest that the development of agricultural strategies be directed to the decrease in pesticide movement. Since soil OM is the chief soil component contributing to the sorption of pesticides and the sorption process influences the magnitude of the other processes and is considered to be one of the major processes affecting the interactions occurring among pesticide and the solid phase in the soil environment [11]. In this study, some solid materials as banana crust meal, biochare and spent tea leaves were used as cost effective adsorbents for elimination of some pesticides from aqueous solution to protect human health.

Methodology:

Tested adsorbents:

Three adsorbent substances were tested in the study. Biochar was obtained from Department of Soil and Water Sciences, Faculty of Agriculture, University of Alexandria. A spent tea leaf was collected and banana crusts were collected, air-dried, ground and passed through a 5-mm sieve. Banana peels from Banana fruits were collected from local market. Banana peels were dried in sunlight for 3 days and cuts into small pieces then washed with distilled water and dried it in 
Citation: Mokhtar S. Beheary, et al., 2018. Removal of Some Pesticides from Contaminated Water Using Low Cost Materials. Advances in Environmental Biology., 12(7): 1-5.

oven at $70^{\circ} \mathrm{C}$ for 5 hours. Oven dried pieces were grounded and sieved using American standard 60 mesh sieves. Spent tea leaves (STL). Black tea from commercial tea bags was used to obtain STL. Tea infusions were first prepared by steeping some tea bags in tap water $\left(125 \mathrm{~mL}\right.$ of water per tea bag) at $90^{\circ} \mathrm{C}$ for 3 min. After this time, STL were recovered and repeatedly washed with distilled water in order to remove soluble and colored compounds. The solid was then rinsed and oven dried at $60^{\circ} \mathrm{C}$ for $24 \mathrm{~h}$. Finally, STL were ground, sieved to $<500 \mu \mathrm{m}$ and stored in polyethylene bags at room temperature until use. Thermal activation of STL was carried out in a muffle furnace by stepwise increase of the temperature (up to $400^{\circ} \mathrm{C}$ ) and isothermal heating for a time of 0.5 to $2 \mathrm{~h}$.

Pesticides:

Table (1) shows the chemical structures of pesticides that are used in the present study.

Table 1: Chemical features of the five pesticides.

\begin{tabular}{|c|c|c|}
\hline Pesticides & Type & Structure \\
\hline Chlorpyrifos & Organophosphate & \\
\hline Dimethoate & Organophosphate & \\
\hline Imidacloprid & Insecticide & \\
\hline Atrazine & $\underline{\text { Herbicide }}$ & \\
\hline Diuron & Algicide and herbicide & \\
\hline
\end{tabular}

Determination of tested pesticides:

Each pesticide standard solutions $(20-100 \mu \mathrm{g} / \mathrm{mL})$ were arranged by the arranged dilution of the pesticide stock solution $(0.5 \mathrm{~g} / \mathrm{L})$. The tested pesticide concentrations in different experiments were examined by a UV-Vis Spectrophotometer (Thermo Corporation, Nicolet, evolution 100). To define the optimum wavelength $\left(\lambda_{\max }\right)$ that is specific for each tested pesticide with smallest interfering, a scanning range of $200-400 \mathrm{~nm}$ was used to create a for $10 \mu \mathrm{g} / \mathrm{mL}$ pesticide solution. The standard calibration curve (C-D curve) was made by plotting triplicates $(n=3)$ against its equivalent absorbance at the optimum obtained $\lambda_{\max }$. For quality assurance and control, triplicate samples containing controls and blanks were done. Standards of pesticide were analyzed with every batch for inter- and intra-batch difference [12].

Adsorption of tested pesticides:

Adsorption isotherms by tested materials were calculated using the batch equilibration method [13]. Trials were done in duplicate with adsorbent mass to pesticide solution 1:5 ratio. The pesticide solutions were equilibrated with the materials in $25-\mathrm{mL}$ polypropylene centrifuge tubes. The tubes were shaken mechanically at $150 \mathrm{rpm}$ at room temperature for $2 \mathrm{~h}$ to achieve equilibrium based on its kinetics study and centrifuged at $4000 \mathrm{rpm}$ for $15 \mathrm{~min}$. The pesticide concentration in supernatants was measured by spectrophotometer instrument at the appropriate $\lambda_{\max }$.

Experimental data are presented as mean \pm standard error and the statistical analysis was performed by the SPSS program (ver. 21.0, USA). 


\section{RESULTS AND DISCUSSION}

Removal of some pesticides from contaminated wastewater has been achieved with varying success. Eliminating some representative types of pesticides as well as atrazine, diuron, imidacloprid, chlorpyrifos and dimethoate from water by biosorbents was researched in this part. Table (2) shows mean removal capacities of biomaterials. In this present study, three adsorbent materials were used for removing Banana crust meal, spent tea leaves and biochare from aqueous solution. The results showed that the removal efficiency by banana crust meal has the order of atrazine > diuron > imidacloprid > dimethoate as a mean percent 99.6, 96.7, 77.9, 64.4 and 61.8, respectively. It was noticed that, high percent of atrazine and diuron was removed by banana crust meal. 14) has used residue materials including orange peels to remove different kinds of PAHs as phenanthrene, naphthalene, acenaphthene, fluorene and pyrene. The results indicated that the removal efficiency by biochare has the order of imidacloprid $>$ atrazine $>$ diuron $>$ chlorpyrifos $>$ dimethoate as a mean percent 93.09, 92.4, 80.1, 72.9 and 42.1, respectively. It was showed that, high percent of atrazine and imidacloprid was removed by biochare. [15] investigated that activated carbon as adsorbent for the removal of herbicide bentazone. [16] investigated the role of apricot stone activated carbon as adsorbent of pesticide depending on adsorbent dose, pesticide concentration, contact time and temperature. Activated carbon that used in the adsorption process is most prevalent and widely used adsorbent in wastewater treatment during the world, but the high charges and regeneration cost of activated carbon limits their large-scale use for the removal of inorganic and organic contaminants, and has encouraged scientists to look for low cost adsorbing materials [17]. The results showed that the removal efficiency by Spent tea leaves has the order of imidacloprid > atrazine > imidacloprid > chlorpyrifos > dimethoate as a mean percent 96.9, 93.74, 82.34, 73.43 and 54.18 respectively. It was showed that, high percent of atrazine and imidacloprid was removed by spent tea leaves. Finally, banana crust meal is the most effective adsorbent for pesticides removal.

Table 2: Removal percentage of tested pesticide residues from water.

\begin{tabular}{|c|c|c|c|c|c|}
\hline \multirow{2}{*}{$\begin{array}{l}\text { Initial conc. } \\
(\mathrm{ppm})\end{array}$} & \multicolumn{5}{|l|}{ \% Removal } \\
\hline & Dimethoate & Chlorpyrifos & Atrazine & Diuron & Imidacloprid \\
\hline \multicolumn{6}{|c|}{ Banana crust meal } \\
\hline 20 & 78.36 & 54.89 & 99.67 & 96.81 & 47.52 \\
\hline 40 & 59.99 & 65.10 & 99.84 & 97.94 & 80.63 \\
\hline 60 & 56.05 & 69.49 & 99.50 & 95.61 & 85.21 \\
\hline 80 & 62.00 & 59.15 & 99.60 & 96.59 & 86.41 \\
\hline 100 & 65.79 & 60.68 & 99.62 & 96.95 & 89.79 \\
\hline Mean & 64.44 & 61.86 & 99.65 & 96.78 & 77.91 \\
\hline \multicolumn{6}{|l|}{ Biochar } \\
\hline 20 & 38.76 & 57.77 & 99.06 & 67.75 & 86.86 \\
\hline 40 & 43.22 & 71.68 & 98.83 & 85.92 & 93.48 \\
\hline 60 & 49.39 & 84.29 & 93.16 & 85.30 & 94.52 \\
\hline 80 & 27.27 & 73.53 & 84.75 & 81.34 & 94.49 \\
\hline 100 & 51.93 & 77.65 & 86.65 & 80.39 & 96.10 \\
\hline Mean & 42.11 & 72.98 & 92.49 & 80.14 & 93.09 \\
\hline \multicolumn{6}{|c|}{ Spent tea leaves } \\
\hline 20 & 70.06 & 52.59 & 86.46 & 84.31 & 94.25 \\
\hline 40 & 28.48 & 77.81 & 92.49 & 84.09 & 97.34 \\
\hline 60 & 43.95 & 78.27 & 96.10 & 88.16 & 97.15 \\
\hline 80 & 62.70 & 80.79 & 96.31 & 83.01 & 97.82 \\
\hline 100 & 65.68 & 77.70 & 97.34 & 72.16 & 97.94 \\
\hline Mean & 54.18 & 73.43 & 93.74 & 82.34 & 96.90 \\
\hline
\end{tabular}

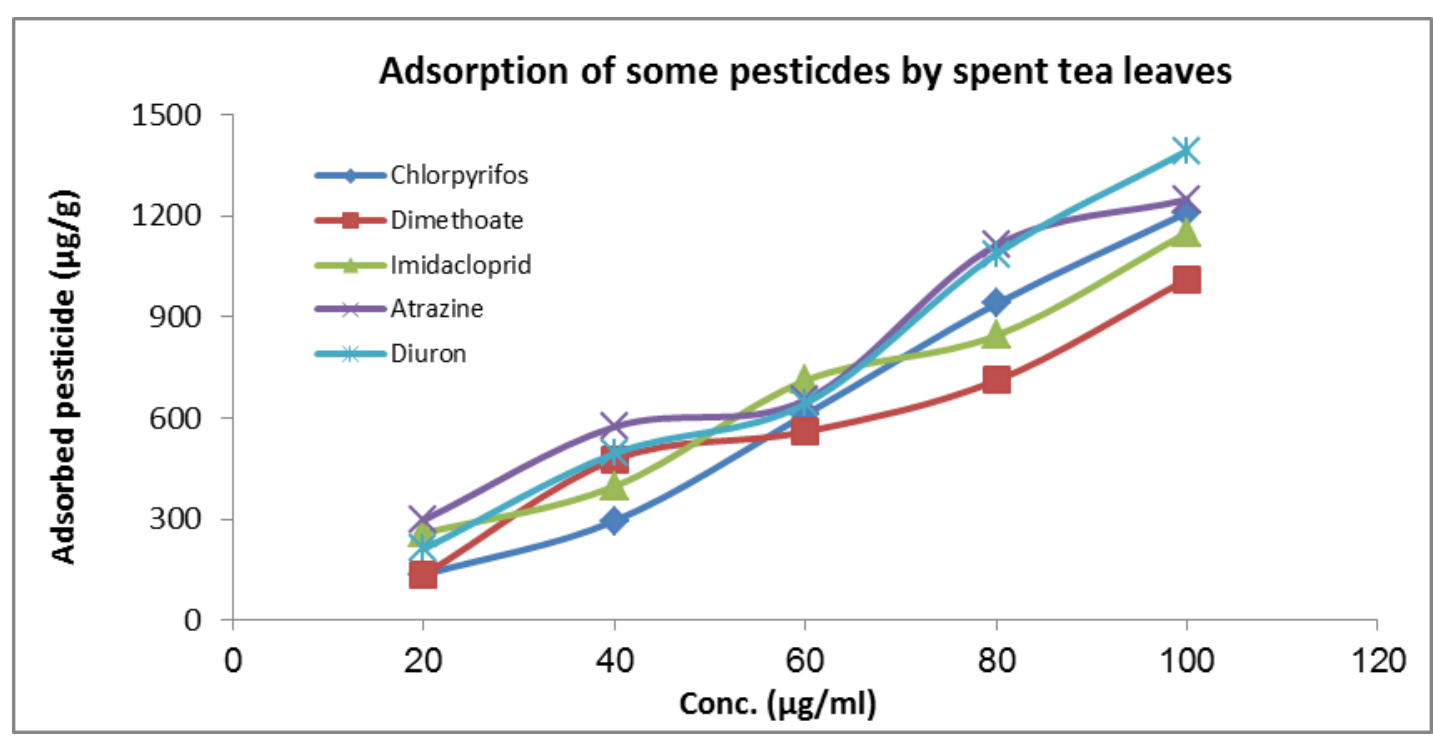



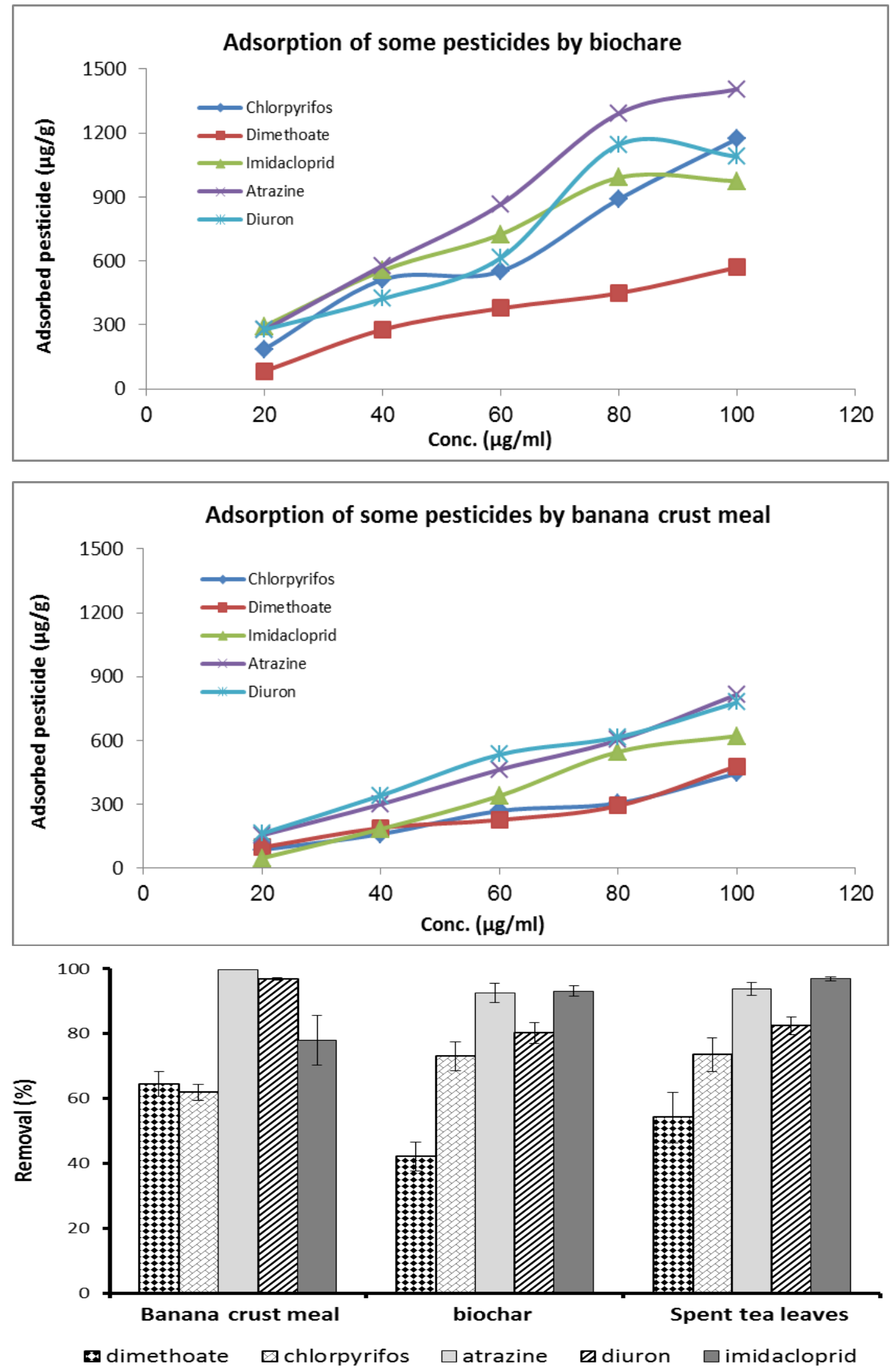

Mean removal percentages $\pm \mathrm{SE}$ of tested pesticides by different materials

Conclusion:

The present study studies the adsorption of some pesticides from contaminated wastewater: atrazine, diuron, imidacloprid, chlorpyrifos and dimethoate. Banana rust meal, biochare and spent tea leave have the ability to remove these toxic pesticides from aqueous solution. Finally, these adsorbents are a more effective and low-cost adsorbents that can be used for the elimination of the studied pesticides and likely other impurities from water. 


\section{REFERENCES}

[1] Vinas, P., N. Campillo, L. Garcia, N. Aguinaga and M.H. Cordoba, 2002. Determination of pesticides in waters by capillary gas chromatography with atomic emission detection. Journal of Chromatography A, 978: 249-256.

[2] Aktar, W., D. Sengupta and A. Chowdhury, 2009. Impact of pesticides use in agriculture: their benefits and hazards, interdisc toxicol Journal, 2: 1-12.

[3] Berny, P. (2007): Pesticides and the intoxication of wild animals. J. Vet. Pharmacol. Ther., 30: 93-100.

[4] Bottoni, P., J. Keizer and E. Funari, 1996. Leaching Indices of some major metabolites. Chemosphere, 32: 1401-1411.

[5] Kumar, M. and L. Philip, 2006. Adsorption and desorption charachteristices of hydrophobic pesticides endosulfan in four Indian soils. Chemosphere. 62: 1064-1077.

[6] Simunek, J., M. Sejna and M.T.H. Van Genuchten, 1999. The hydrus- 2D software pac Kage for simulating the two dimension movement of water, heat and multiple solutes in variably- saturated media.

[7] Gao, J.P., J. Maguhn, P. Spitzauer and A. Kettrup, 1998. Sorpotion of pesticides in the sediment of the Teufelsweiher pond, 32: 1662-1672.

[8] Navarro, M.C., C. Pérez-Sirvent, M.J. Martínez-Sánchez, J. Vidal, P.J. Tovar and J. Bech, 2008. Abandoned mine sites as a source of contamination by heavy metals: A case study in a semi-arid zone. Journal of Geochemical Exploration, 96: 183-193.

[9] Hantush, M.M., R. Islam and M.A. Marño, 2000. Models for soil-aquifer vulnerability assessment and pollution prevention. J. Hydrol., 227: 66- 80.

[10] Leistra, M., A.M.A. van der Linden, J.J.T.I. Boesten, A. Tiktak and F. Van den Berg, 2001. PEARL model for pesticide behaviour and emissions in soilplant systems. Descriptions of the processes in FOCUS PEARL v 1.1.1. Alterra-Rapport 013, RIVM report 711401009. Alterra Wageningen UR, Wageningen / National Institute of Public Health and the Environment (RIVM), Bilthoven.

[11] Khan, S.U., 1978. The interaction of organic matter with pesticides. Developments in soil science, 8: 137-171.

[12] Jan, M.R, J. Shah, M. Muhamad and B. Ara, 2009. Glyphoate herbicide residue determination in samles of environmental importance using spectrophotometric method. J Haz Mat., 169: 742-745.

[13] Walker, A. and S.J. Welch, 1989. The relative movement and persistence in soil of chlorsulfuron, metsulfuron-methyl and triasulfuron. Weed Research, 29: 375-383.

[14] Khan, S.U., 1978. The interaction of organic matter with pesticides. Developments in soil science, 8: 137-171.

[15] Njoku, V.O., M.D. Azharul Islam, M. Asif and B.H. Hameed, 2014. Utilization of sky fruit husk agricultural waste to produce high quality activated carbon for the herbicide betazon adsorption. Chemical Engineering Journal, 251: 183-191.

[16] Mohammed, H.J., B.J. Kadhim and A.S. Mohammed, 2011. Adsorption study of some sulphanilic azo dyes on charcoal, E-J. Chem., 8: $739-747$.

[17] Bottoni, P., J. Keizer and E. Funari, 1996. Leaching Indices of some major metabolites. Chemosphere, 32: 1401-1411. 